\title{
Vein of Galen malformation treated with the Micro Vascular Plug system: case report
}

\author{
Woojin Joo, BS, ${ }^{1}$ Philippe Mercier, MD, PhD, ${ }^{1}$ Shiva Kheradmand, BS, ${ }^{2}$ Peyman Shirani, MD, ${ }^{1}$ \\ Ahmed Sarhan, MD,' Samer K. Elbabaa, MD,'1 and Randall C. Edgell, MD'1 \\ ${ }^{1}$ St. Louis University School of Medicine, St. Louis, Missouri; and 2Midwestern University Arizona College of Osteopathic \\ Medicine, Glendale, Arizona
}

\begin{abstract}
This case report describes the use of the Micro Vascular Plug (MVP) system in a mural-type vein of Galen aneurysmal malformation, resulting in successful occlusion of the cerebrovascular fistula. This result was confirmed with immediate cessation in blood flow and 6-month follow-up angiography. The unique characteristics of the MVP provide potential advantages over current embolic techniques for occlusion of high-flow, fistulous, intracranial vessels such as those seen in the vein of Galen aneurysmal malformation, and may represent a valuable addition to the currently available options.
\end{abstract}

https://thejns.org/doi/abs/10.3171/2016.12.PEDS16305

KEY WORDS arteriovenous malformation; endovascular; Micro Vascular Plug; vein of Galen; vascular disorders

$\mathrm{T}$ HE vein of Galen aneurysmal malformation (VGAM) is a rare arteriovenous shunt that is estimated to represent $30 \%$ of all pediatric vascular malformations. ${ }^{8}$ It is a lesion supplied by the choroidal arteries through a fistulous connection with the median vein of the prosencephalon, the embryonic precursor of the vein of Galen. The venous anatomy seen in the VGAM is similar to that seen in the 12th gestational week of embryonic development, suggesting that the VGAM is a result of an error in vasculogenesis during this time period. The VGAM can be dichotomized into the mural type (one or more direct arteriovenous fistulas within the wall of the median vein of the prosencephalon) and the choroidal type (multiple arterial feeders creating a niduslike structure that shunts into the dilated venous pouch). Clinical manifestations differ significantly depending on which angioarchitecture is present, with the mural type associated with less severe symptoms and an older age at presentation. ${ }^{1}$

The most common causes of morbidity and mortality are high-output congestive heart failure (most common neonatal presentation), hydrocephalus (most common presentation in infants), headache, and seizures. Left untreated, VGAM is associated with high rates of morbidity and mortality. ${ }^{3}$ Over the last 3 decades, endovascular treatments have dramatically improved the prognosis. There is the potential for cure, and up to $74 \%$ of treated patients have normal neurological profiles at long-term follow-up. ${ }^{1}$ The techniques have evolved over time to include coils, $\mathrm{N}$-butyl cyanoacrylate (TruFill; Codman Neuro) and ethylene vinyl alcohol copolymer (Onyx; Medtronic, Inc.). ${ }^{3,12}$ The Micro Vascular Plug (MVP; Medtronic, Inc.) was recently introduced for arterial occlusion and has been used with promising results in neurovascular disease..$^{2,9,14} \mathrm{To}$ 
our knowledge, this is the first reported case in which the MVP was used for treatment of VGAM.

\section{Case Report}

\section{History and Examination}

A 4-month-old girl with no significant birth history initially presented to her pediatrician with symptoms of nasal and sinus congestion. These symptoms worsened despite medical therapy, progressing to greater congestion, gasping for air during sleep, decreased feeding, and lethargy. Physical examination was significant for increased head circumference, full fontanelles, distended scalp veins, minimal spontaneous movement, and lethargy. A CT scan of the head was performed, and showed ventriculomegaly and a large "pineal region mass" ( $4.4 \times$ $3.3 \times 4.8 \mathrm{~cm}$ ) (Fig. 1).

\section{Neuroimaging Studies}

The patient was hospitalized. Results of an MRI study of the brain were consistent with a VGAM with a stenotic segment of the median promesencephalic vein followed by dilation of the confluence of sinuses. Hydrocephalus was thought to be caused by high venous pressures and venous hypertension in addition to mass effect of the malformation on the cerebral aqueduct. The combination of the patient's clinical signs of lethargy and gasping breaths, a concern for further exacerbation by increased intracranial pressure while she was supine for a lengthy procedure, and the anticipation that the mass effect produced by the malformation on the cerebral aqueduct would take months to resolve despite occlusion of the fistula prompted a decision to insert a ventriculoperitoneal shunt prior to embolization. A Strata valve with an initial setting at 2.0 was used to prevent rapid decompression of the ventricu-

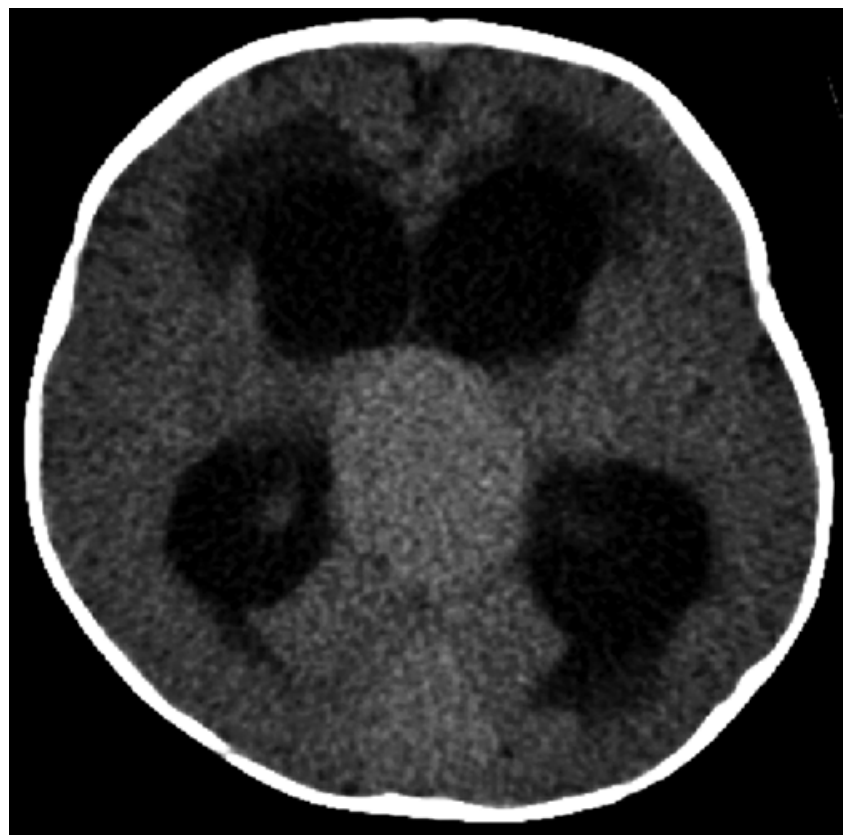

FIG. 1. Axial CT scan obtained prior to embolization, showing ventriculomegaly, transependymal edema, and a "pineal region mass." lar system, which has been thought to be associated with neurological decline following CSF shunt placement in the setting of VGAM. . $^{1,713}$

A catheter angiogram showed that the VGAM was supplied by an enlarged left lateral posterior choroidal artery, with a fistulous connection to the median vein of the prosencephalon and draining into a dilated embryonic falcine sinus (Fig. 2). In addition, an echocardiogram showed mild high-output congestive heart failure with preserved systolic function, probably secondary to the VGAM.

\section{Treatment}

Intervention took place with the aid of biplane angiography. A 5-Fr sheath was placed in the right femoral artery and a 5-Fr Envoy (Codman Neuro) guide catheter was navigated into the aortic arch. It was used to select the left common carotid artery, and then it was advanced into the left internal carotid artery. A 0.021-inch Prowler Plus Select (Codman Neuro) microcatheter and a Synchro 14 microwire (Stryker Neurovascular) were navigated intracranially into the fetal posterior cerebral artery, followed by the lateral posterior choroidal artery. An MVP-3 device was deployed within the feeding artery. Immediately after deployment, repeat angiography showed no flow through the fistulous connection (Fig. 3).

\section{Posttreatment Outcome and Follow-Up}

A 24-hour follow-up CT scan showed thrombosis of the VGAM (Fig. 4). Over the next 9 months, the patient demonstrated steady clinical improvement, with her head circumference returning to the normal range and normal developmental progression. At her 9-month follow-up, she was meeting all her development milestones and her

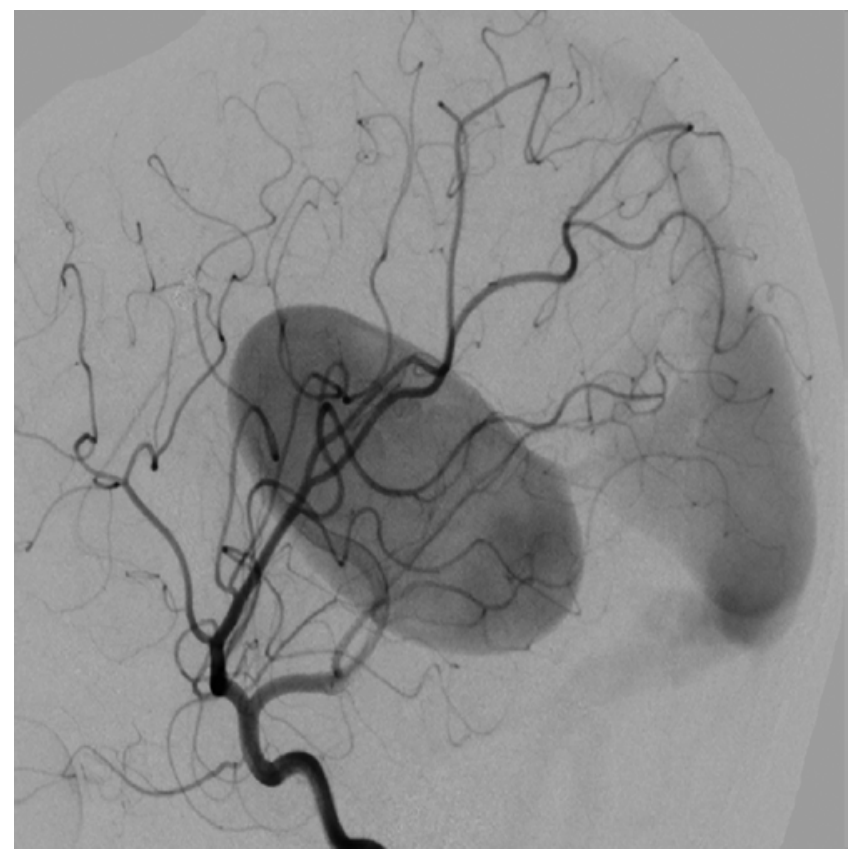

FIG. 2. Digital subtraction angiography study obtained prior to embolization confirming VGAM, with the posterior choroidal artery feeding into the ectatic vein. 


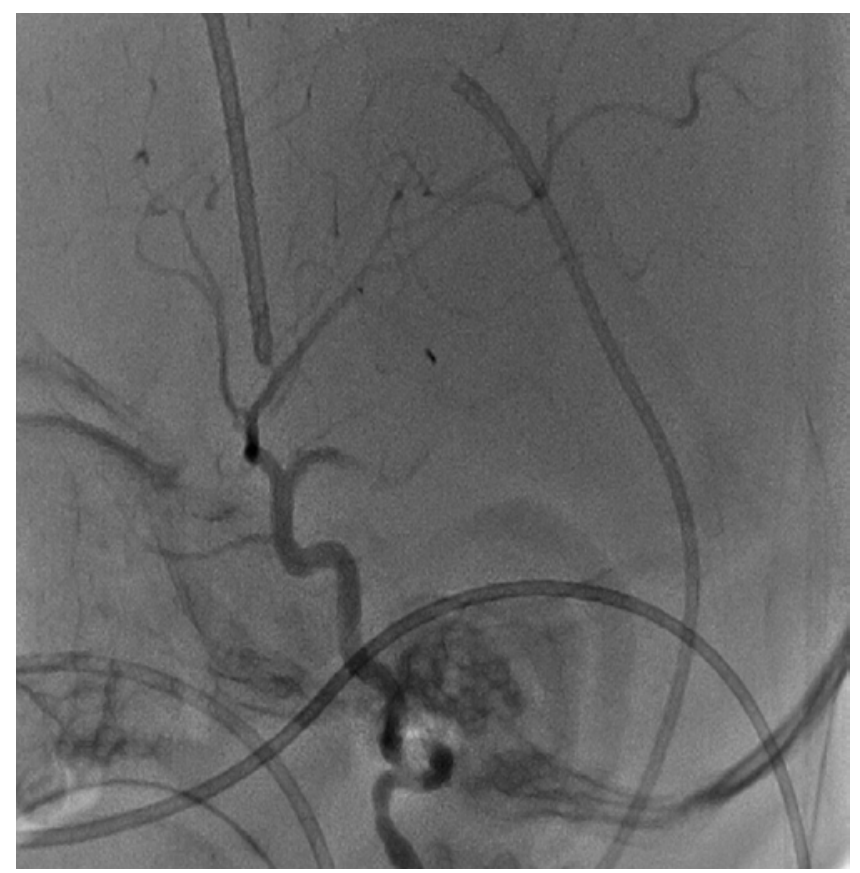

FIG. 3. Digital subtraction angiography study obtained immediately after embolization with the MVP.

head circumference was down to the 88th percentile for her age. Follow-up angiography at 6 months postembolization showed complete resolution of the VGAM. Serial MRI sessions, including one prior to embolization, one at 3 months, and one at 6 months postembolization, demonstrated gradual reduction in the size of the dilated VGAM and eventual decompression of the aqueduct (Fig. 5). We suspected that by her 9-month follow-up visit, the patient was no longer dependent on her ventriculoperitoneal shunt. With regard to her cardiac function, a transthoracic echocardiogram obtained 3 weeks after embolization showed the cardiac structure and physiology to be within normal limits.

\section{Discussion}

There has been a gradual but steady evolution in the endovascular treatment of the VGAM..$^{6,10,15,16}$ Liquid embolic agents have been the preferred modality of treatment for several decades-initially with $N$-butyl cyanoacrylate and more recently also with ethylene vinyl alcohol copolymer. These agents have been combined with dual-lumen balloon catheters or detachable-tip catheters to enhance the safety of infusion. We report the successful use of the MVP system in a patient with VGAM, and we discuss the potential role of this new device in this population.

The MVP system consists of a wide-meshed, nitinol, ovoid frame that is partially covered on its proximal surface with a polytetrafluoroethylene membrane. It has radiopaque markers at the proximal and distal ends of the device, and is attached to a pusher wire. The device comes in 2 sizes: MVP-3 (maximum diameter $5.3 \mathrm{~mm}, 0.21$-inch microcatheter) and MVP-5 (maximum diameter $6.5 \mathrm{~mm}$, 0.27 -inch microcatheter). Once the device is delivered through the microcatheter to the site of deployment, pull-

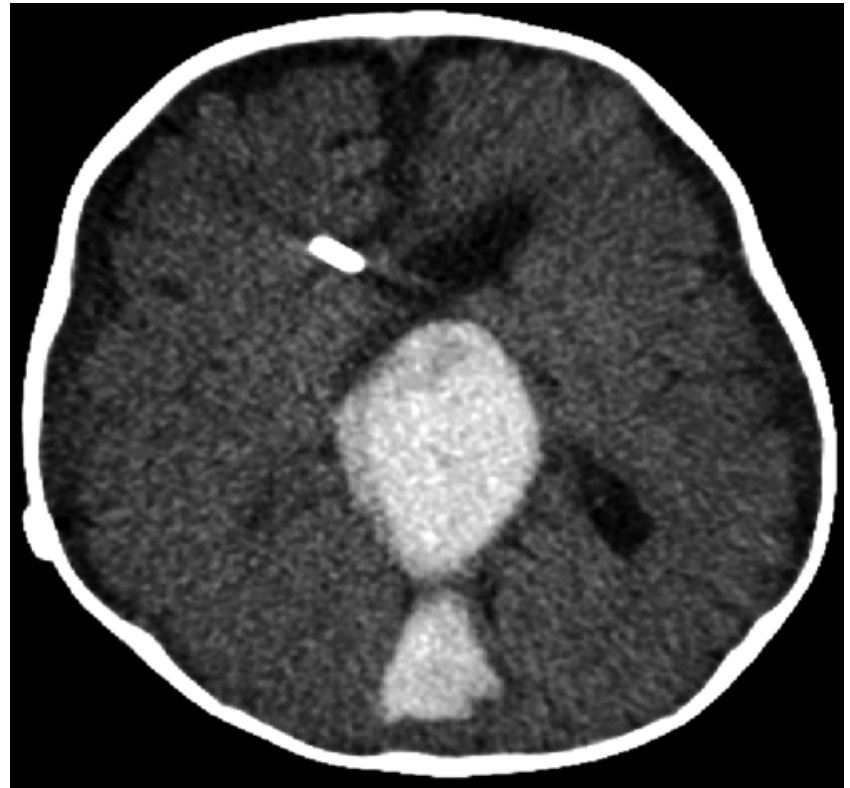

FIG. 4. Axial CT scan obtained within 24 hours after embolization with the MVP device, showing thrombosis of the VGAM.

ing back the catheter unsheathes it. It can be recaptured if needed, but if a satisfactory position is obtained the MVP can be mechanically detached by rotating the pusher wire (Fig. 6).

The use of liquid embolic agents for transarterial treatment of patients with VGAM was a significant step forward, but is not without limitations., ${ }^{4,6,12}$ Their use in highflow vessels is challenging due to the tendency of these agents to diffuse distally into the venous system and/or into normal arterial branches. Attempts to mitigate this risk have included the following: coil placement in the arterial feeder prior to liquid embolic injection, retrograde catheterization of arterial feeders through the venous side prior to injection, and use of adenosine to induce cardiac standstill during injection. ${ }^{12}$ Pop and coworkers further reported their own attempts at flow control by using a duallumen balloon microcatheter prior to liquid embolization. By inflating the balloon at the tip of this microcatheter, they were able to slow the flow of blood sufficiently to embolize the arterial pedicle without significant migration of ethylene vinyl alcohol copolymer into the venous system. This technique does carry a risk of balloon-induced vessel rupture, however. ${ }^{12}$ Microcoils, on the other hand, provide a targeted and controlled vessel occlusion. However, their use in the treatment of VGAM is not ideal due to the need for numerous coils in a high-flow region, increasing procedure and anesthetic time as well as cost. ${ }^{2}$

The MVP system may have several advantages in the appropriate patient. The compatibility of the device with microcatheters capable of intracranial navigation is an important advance over other vascular occlusion devices currently on the market. ${ }^{2}$ The MVP offers greater precision in the site of occlusion relative to liquid embolic agents; distal migration appears to be rare in appropriately sized vessels. ${ }^{2,5,10,15}$ The device can also be resheathed if the initial location of deployment is not ideal. The occlusive effect is rapid relative to coil embolization, with com- 

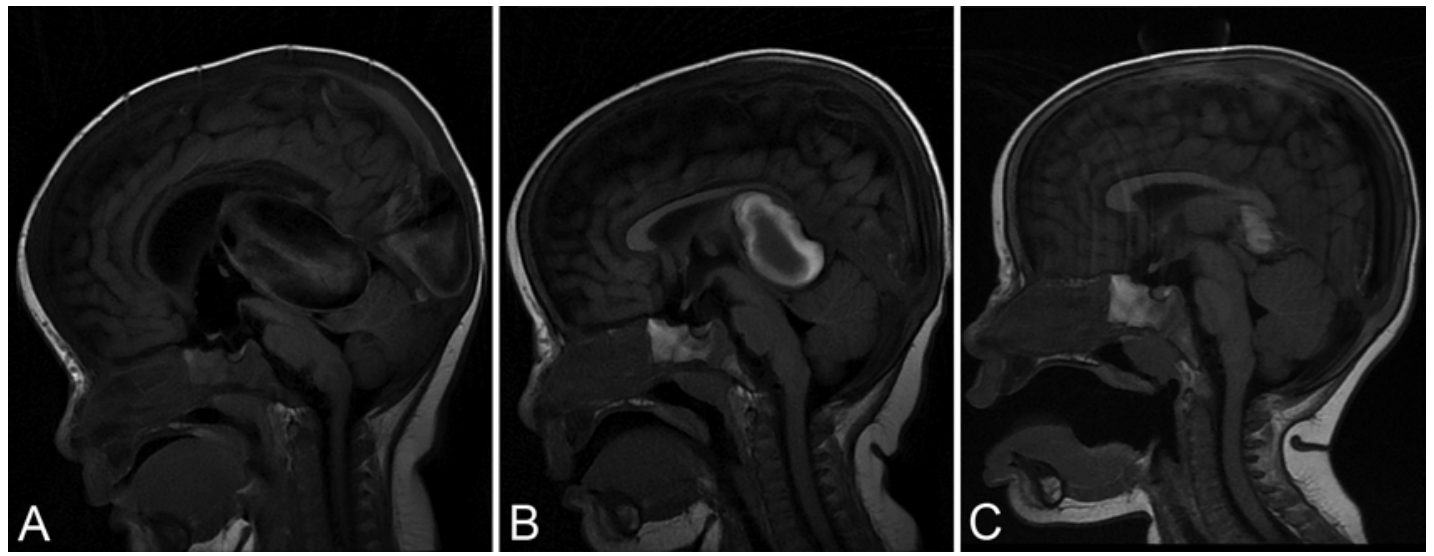

FIG. 5. Sagittal T1-weighted MRI studies obtained without contrast prior to embolization (A), at a 3-month follow-up visit (B), and at a 6 -month follow-up visit (C), demonstrating gradual reduction in the size of the fistula and resolution of the mass effect on the cerebral aqueduct.

plete occlusion occurring in less than 2 minutes in one series. ${ }^{2}$ Finally, there may be a cost advantage in cases of mural VGAM with a limited number of arterial pedicles. Although long-term follow-up studies are needed, our patient, as well as those in reports by other authors, show promising results. ${ }^{5,9,11,14}$

There are several limitations to the MVP system. The range of vessel diameters that can be safely treated is 1.5 $5 \mathrm{~mm}$, and the device must be appropriately sized. ${ }^{14} \mathrm{Ad}$ ditionally, the device requires a landing zone of $12-15 \mathrm{~mm}$ for effective deployment. It is theoretically possible that proximal deployment, necessitated by the lack of a distal landing zone, would allow recruitment of adjacent arteries and reperfusion of the lesion. ${ }^{5}$ The compatible microcatheters, although capable of intracranial catheterization, are not ideal when navigating the highly tortuous and small vessels of patients with VGAM. Finally, there has been one report of premature detachment of the MVP, which could have catastrophic consequences in the wrong location. ${ }^{10}$

\section{Conclusions}

We report the successful use of the MVP system to treat a patient with a mural-type VGAM, with complete

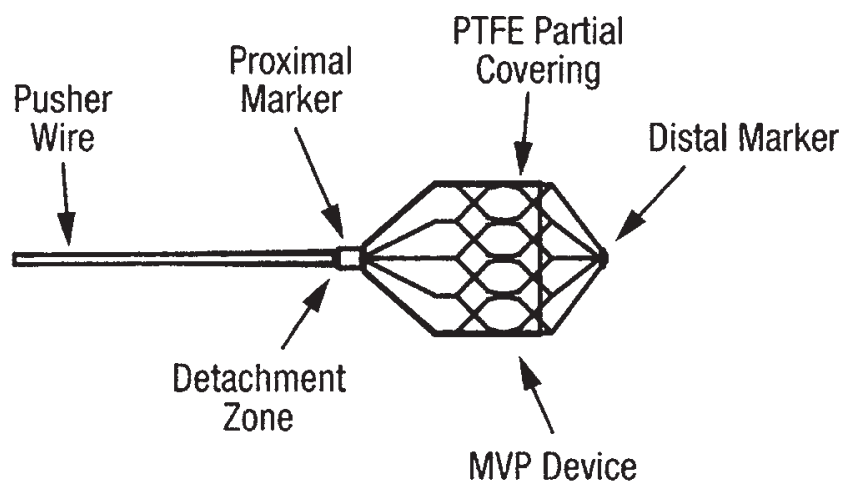

FIG. 6. Schematic depiction of the MVP system. PTFE = polytetrafluoroethylene. Image courtesy of Medtronic. (C) Medtronic. All rights reserved. cure at 6-month follow-up. To our knowledge, this is the first such case reported in the medical literature. The use of the MVP system may augment the currently available therapeutic options for the VGAM in the appropriately selected patient and warrants further investigation with long-term follow-up.

\section{References}

1. Alvarez H, Garcia Monaco R, Rodesch G, Sachet M, Krings T, Lasjaunias P: Vein of Galen aneurysmal malformations. Neuroimaging Clin N Am 17:189-206, 2007

2. Beaty NB, Jindal G, Gandhi D: Micro Vascular Plug (MVP)-assisted vessel occlusion in neurovascular pathologies: technical results and initial clinical experience. J Neurointerv Surg 7:758-761, 2015

3. Berenstein A, Fifi JT, Niimi Y, Presti S, Ortiz R, Ghatan S, et al: Vein of Galen malformations in neonates: new management paradigms for improving outcomes. Neurosurgery 70:1207-1214, 2012

4. Berenstein A, Ortiz R, Niimi Y, Elijovich L, Fifi J, Madrid $\mathrm{M}$, et al: Endovascular management of arteriovenous malformations and other intracranial arteriovenous shunts in neonates, infants, and children. Childs Nerv Syst 26:1345-1358, 2010

5. Conrad MB, Ishaque BM, Surman AM, Kerlan RK Jr, Hope MD, Dickey MA, et al: intraprocedural safety and technical success of the MVP micro vascular plug for embolization of pulmonary arteriovenous malformations. J Vasc Interv Radiol 26:1735-1739, 2015

6. Hoang S, Choudhri O, Edwards M, Guzman R: Vein of Galen malformation. Neurosurg Focus 27(5):E8, 2009

7. Jea A, Bradshaw TJ, Whitehead WE, Curry DJ, Dauser RC, Luerssen TG: The high risks of ventriculoperitoneal shunt procedures for hydrocephalus associated with vein of Galen malformations in childhood: case report and literature review. Pediatr Neurosurg 46:141-145, 2010

8. Johnston IH, Whittle IR, Besser M, Morgan MK: Vein of Galen malformation: diagnosis and management. Neurosurgery 20:747-758, 1987

9. Kleine JF, Prothmann S, Boeckh-Behrens T: Occlusion of small arteries in the neuroendovascular and head and neck territory-initial experiences with a microvascular plug. J Vasc Interv Radiol 26:426-431, 2015

10. Lasjaunias P, Garcia-Monaco R, Rodesch G, terBrugge K, Zerah M, Tardieu M, et al: Vein of Galen malformation. 
Endovascular management of 43 cases. Childs Nerv Syst 7:360-367, 1991

11. Pellerin O, Maleux G, Déan C, Pernot S, Golzarian J, Sapoval M: Microvascular plug: a new embolic material for hepatic arterial skeletonization. Cardiovasc Intervent Radiol 37:1597-1601, 2014

12. Pop R, Manisor M, Wolff V, Kehrli P, Marescaux C, Beaujeux R: Flow control using Scepter ${ }^{\mathrm{TM}}$ balloons for Onyx embolization of a vein of Galen aneurysmal malformation. Childs Nerv Syst 31:135-140, 2015

13. Schneider SJ, Wisoff JS, Epstein FJ: Complications of ventriculoperitoneal shunt procedures or hydrocephalus associated with vein of Galen malformations in childhood. Neurosurgery 30:706-708, 1992

14. Shwe Y, Paramasivam S, Ortega-Gutierrez S, Altschul D, Berenstein A, Fifi JT: High-flow carotid cavernous fistula and the use of a microvascular plug system: initial experience. Interv Neurol 3:78-84, 2015

15. Tiwary S, Geethanath RM, Abu-Harb M: Vein of Galen malformation presenting as persistent pulmonary hypertension of newborn (PPHN). BMJ Case Rep 2013 [epub ahead of print], 2013

16. Yan J, Wen J, Gopaul R, Zhang CY, Xiao SW: Outcome and complications of endovascular embolization for vein of Ga- len malformations: a systematic review and meta-analysis. $\mathbf{J}$ Neurosurg 123:872-890, 2015

\section{Disclosures}

The authors report no conflict of interest concerning the materials or methods used in this study or the findings specified in this paper.

\section{Author Contributions}

Conception and design: all authors. Acquisition of data: all authors. Analysis and interpretation of data: all authors. Drafting the article: all authors. Critically revising the article: all authors. Reviewed submitted version of manuscript: all authors. Approved the final version of the manuscript on behalf of all authors: Edgell. Administrative/technical/material support: all authors. Study supervision: all authors.

\section{Correspondence}

Randall C. Edgell, St. Louis University School of Medicine, 3635 Vista Ave., St. Louis, MO 63110. email: redgell@slu.edu. 